\title{
OPTIMUM BARITT STRUCTURE
}

\author{
S. LURYI and R. F. KAZARINOV \\ Bell Laboratories, Murray Hill, NJ 07974, U.S.A.
}

(Received 22 February 1982; in revised form 19 March 1982)

\begin{abstract}
The recently studied planar-doped triangular barrier structure is analyzed theoretically for a possible application as a barrier injection transit time device (BARITT). The small signal impedance as well as the large-signal power characteristics are calculated. Compared to the conventional BARITT structures the new device offers a significant improvement in its frequency range and generated power.
\end{abstract}

The idea of two-terminal devices with a negative dynamical resistance arising from the transit-time effect in semiconductor structures was first considered by Shockley in 1954. A number of devices have been since proposed and implemented based on this principle (Ref. [1], Chap. 10), the most important of those being the IMPATT and BARITT diodes. All transit time devices contain a high-field region in which carriers travel at saturated velocity and develop a charge density wave which contributes to a phase delay between the ac current and voltage. For physical reasons this delay cannot exceed $90^{\circ}$ and therefore one needs an additional delay to achieve a negative resistance. Such initial phase shift can be obtained from an input element which injects carriers into the high-field transit-time region.

In IMPATT diodes, injection occurs due to avalanche breakdown in a reverse-biased $p-n$ junction. In BARITT structures the injection mechanism is provided by thermionic emission over a barrier. The avalanche injection is equivalent to an LC input circuit without significant active resistance, which creates a $\pi / 2$ phase delay. The thermionic injection mechanism corresponds to an RC circuit which contributes a positive (unwelcome) active resistance and gives rise to a delay which depends on the parameters of the structure. Because of this the BARITT diode operates at lower power and lower efficiency than the IMPATT diode. On the other hand the noise associated with thermionic injection over a barrier is significantly lower than the avalanche noise in an IMPATT diode.

In its original version the BARITT represents a reachthrough $n p n$ (or $p n p$ ) diode[1]. In the working state of the device the central $p$ (or $n$ ) layer is depleted of carriers giving rise to a potential barrier for thermionic injecting. The downhill portion of this barrier works as a base and the uphill portion as an injector. The flat region near the top introduces parasitic positive resistance. Various multilayer structures have been proposed, e.g. nippn structure in Ref. [2], in order to reduce this resistance and thus improve the power and efficiency characteristics. The optimum limit of the BARITT corresponds to a structure in which the central layer is ultra-thin, i.e. represents a charge sheet. Such structures (planar-doped by MBE $\left.n-i-p^{+}-i-n\right)$ have been recently studied experimentally $[3,4]$ and theoretically $[5]$. Fortunately, it is precisely in the charge-sheet limit that the structure admits of a rigorous analytical theory. In Ref. [5] the planar-doped diode was analyzed in a static regime. In the present work we analyze its high-frequency characteristics and investigate its potential application as a BARITT device.

The structure, shown in Fig. 1, includes a nearly intrinsic (i) semiconductor layer sandwiched between two $n^{+}$doped layers. In the process of growth of the structure by $\mathrm{MBE}$ a thin $p^{+}$layer is built in the $i$ region. All acceptors in this layer are ionized forming a negatively charged sheet which gives rise to a potential barrier of triangular shape. For the transit time performance the triangular barrier (TB) must be strongly asymmetric, that is $L_{1} \ll L_{2}$. The dc voltage is applied with + to the longer side so that the downhill slope, $L_{2}$, plays the role of a high-field transit-time region and the uphill side, $L_{1}$, that of an injector.

The small-signal impedance of the base is calculated in the same way as in the conventional BARITT theory (Ref. [1], p. 619) and is given by $Z(\omega)=R-i X$ with

$$
\begin{gathered}
R=\frac{L_{2} \cos \alpha}{\epsilon \omega \theta}[\cos \alpha-\cos (\alpha+\theta)] \\
X=\frac{L_{2}}{\omega \epsilon}\left\{1+\frac{\cos \alpha}{\theta}[\sin \alpha-\sin (\alpha+\theta)]\right\}
\end{gathered}
$$

where $\theta \equiv \omega L_{2} / v_{s}$ is the transit time angle at frequency $\omega, v_{s}$ saturation velocity, $\epsilon$ the dielectric permittivity, and $\alpha$ the injection phase delay.

The form of eqn (1) corresponds to the characteristic BARITT boundary condition which relates the ac particle current density $\delta J$ to the ac field $\delta E$ at the injection plane (which in our case is at the top of the barrier), viz.

$$
\delta J\left(L_{1}\right)=\sigma \delta E\left(L_{1}\right)
$$

where $\delta$ is related to $\alpha$ of eqn (1) by

$$
\tan \alpha=\omega \in / \sigma .
$$

The boundary condition, eqn (2) describes an equivalent 


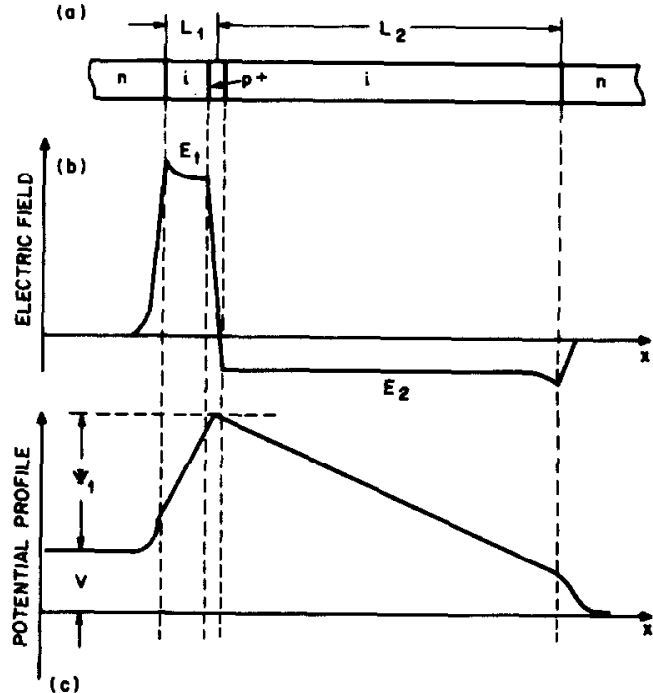

Fig. 1. Schematic diagram of a T-BARITT structure (a), electric field (b), and electrostatic potential (c) distributions under a d.c. bias.

$R C$ circuit with a time constant $R_{\text {in }} C_{\text {in }}=\epsilon / \sigma$. Another form of eqn (2) can be given in terms of the total ac current $\delta I$ as follows:

$$
\delta J\left(L_{1}\right)=\cos \alpha \mathrm{e}^{-i \alpha} \delta I
$$

where $\delta I$ includes the displacement current.

The barrier conductance $\sigma$ per unit area can be found from the static theory of charge injection over triangular barriers [5]. Equations (5) and (6) of Ref. [5] give an expression of the particle current density $J$ in terms of the asymptotic field $E_{1}$ on the uphill side of the $T B$. Using these equations and the definition eqn (2) of $\sigma$ we find

$$
\sigma=\frac{J_{a}}{k T} \frac{\partial \psi_{1}}{\partial E}=-\frac{J_{a}}{k T} \frac{\partial \psi_{1}}{\partial\left|E_{1}\right|}
$$

where

$$
\begin{aligned}
\psi_{1}\left(E_{1}\right)=- & \left|E_{1}\right| L_{1}+\frac{2 k T}{q} \ln 2 \\
& E_{1}\left[\left(1+\epsilon_{1}{ }^{2} \mathrm{e}^{1+\epsilon_{1}^{2}}\right)^{1 / 1}-\left(\epsilon_{1}{ }^{2} \mathrm{e}^{1+\epsilon_{1}^{2}}\right)^{1 / 2}\right]
\end{aligned}
$$

with $\epsilon_{1}=E_{1}{ }^{2} / 4 k T N_{d}$ and $N_{d}$ being the donor concentration in the injector $n^{+}$layer. The quantity $\tilde{L}_{1} \equiv \partial \psi_{1} / \partial E$ represents an effective length of the barrier shoulder on the injecting side. At low currents, when $\epsilon_{1} \geq 1$, one has $\tilde{L}_{1} \sim L_{1}$ to within the Debye length in the $n^{+}$material. In the high current limit, when $E_{1} \ll 1$ we have

$$
\tilde{L}_{1}=L_{1}-2 k T / q E_{1} \text {. }
$$

The effective length $\tilde{L}_{1}$ decreases with increasing current because of the diffusion of carriers. It is important to understand the limits of validity of eqns (5) and (6). Clearly, $\tilde{L}_{1}$ given by eqn (7) must remain positive. In- deed, eqn (6) was derived in [5] under conditions which are equivalent to the assumption $\tilde{L}_{1}>L_{\mathrm{D}}$. The corresponding restriction on the current density $J$ in eqn (5) is given by $J<J_{\max }$ where

$$
J_{\max }=A^{*} T^{2}\left(L_{D} / L_{1}\right)^{2}
$$

where $A^{*}$ is an effective Richardson constant (Ref. [1], Chap. [5]).

As seen from eqn (1a) the active resistance $R$ of the drift region can take negative values. On the other hand the injection region introduces, in series, a positive active resistance, $r$, which corresponds to the real part of the impedance of the equivalent $R C$ input circuit. We can find $r$ from the known time constant $R_{i n} C_{i n} \equiv \epsilon / \sigma$ of the injection region. Indeed, the differential capacitance of this region is given by $C_{\ln } \equiv \epsilon\left(\partial \psi_{1} / \partial E\right)^{-1}=\epsilon / \tilde{L}_{1}$, whence we have

$$
r=\tilde{L}_{1} \sin \alpha \cos \alpha / \epsilon \omega .
$$

The total active resistance of the device, $R_{\mathrm{tot}}=R+r$, is given by the sum of eqns (1a) and (9). The negative $R_{\text {tot }}$ reaches its maximum,

$$
R_{\mathrm{tot}}=\frac{1}{8 \pi \epsilon \omega}\left[L_{2}-2 \pi \sqrt[V]{ }(3) \tilde{L}_{1}\right]
$$

at the following values of the parameters: $(\alpha+\theta)=2 \pi$ and $\alpha=\pi / 3$. These values of $\theta$ and $\alpha$ correspond to

$$
L_{2}=5 \pi v_{s} / 3 \omega
$$

and

$$
J=k T \omega \in / V(3) q \tilde{L}_{1} .
$$

The required condition eqn (11b) on the current density falls within the limits of validity of our theory expressed by eqn (8) provided that the angular frequency $\omega$ does not exceed a maximum value given by

$$
\omega_{c}=\left(N_{c} / N_{d}\right) \bigvee(3) v_{R} \tilde{L}_{1} / L_{1}^{2}
$$

where $v_{R} \equiv\left(k T / 2 \pi m^{*}\right)^{1 / 2}=A^{*} T^{2} / q N_{c}$ is the mean thermal velocity of electrons in a given direction (Ref. [1], Chap. 5). In silicon, for $N_{d} \sim 10^{18} \mathrm{~cm}^{-3}$, i.e. $L_{D}=$ $40 \AA$, taking $L_{1} \sim 300 \AA$ and $\tilde{L}_{1} \sim 100 \AA$ one finds from eqn (12) that $\omega_{c} \sim 3 \times 10^{13} \mathrm{sec}^{-1}$. This means that for all reasonable operating frequencies the conditions (11) which optimize the negative resistance can be fulfilled. Of course, the actual operating frequency is determined by the time of flight of electrons across the drift region according to eqn (11a) and its only theoretical limitation is due to the condition $L_{2}>2 \pi V(3) \tilde{L}_{1} \geqslant 1000 \AA$ which comes from eqn (10). This implies that the triangular barrier device (T-BARITT) can operate at frequencies $f=\omega / 2 \pi$ up to $f_{\max } \sim 1000 \mathrm{GHz}$.

We now turn our attention to the power characteristics. To our knowledge there is no rigorous large signal theory of transit time devices. However, the existing 
approximate theories give a reasonable account of the experimental situation. The standard approach [6-8] is based on the analysis of the dominant nonlinearity in a device, such as field dependence of the ionization coefficient in the IMPATT [7] or exponential currentvoltage dependence, characteristic of the thermionic emission process in the BARITT[8]. For example, in Ref. [8] it was assumed that in a large signal operation of a BARITT the barrier height oscilates sinusoidally with an amplitude equal $50 \%$ of its average value. A similar analysis for the T-BARITT is simplified by the observation that even at the highest frequencies, $f \sim f_{\max }$, the residual average barrier height $\left\langle\psi_{1}\right\rangle$ corresponding to the bias chosen so as to fulfill the condition (11b), is still large compared to $k T / q$. The barrier height, $\psi_{1}(t)=$ $\left\langle\psi_{1}\right\rangle+\Delta \psi_{1}(t)$, oscillates periodically giving rise to a periodic variation of the particle current $J(t) \propto$ $\exp \left[\beta \Delta \psi_{1}(t)\right]$. Assuming, following [8], a sinusoidal oscillation, $\Delta \psi_{1}(t)=1 / 2\left\langle\psi_{1}\right\rangle \cos \omega t$ we can expand $J(t)$ in a Fourier cosine series

$$
J(t)=\text { const }\left[I_{0}\left(\frac{1}{2} \beta\left\langle\psi_{1}\right)\right)+I_{1}\left(\frac{1}{2} \beta\left\langle\psi_{1}\right\rangle\right) \cos \omega t\right]
$$

where $I_{0}$ and $I_{1}$ are the modified Bessel functions. The higher order harmonics omitted in the expansion (13) contribute only a negligible power dissipation because of the filtering properties of an external contour. Indeed, in a microwave generator the external circuit represents an $\mathrm{LC}$ resonance filter which suppresses the losses at nonresonant frequencies because of a large reactive part of its impedance. Now, since $\beta\left(\psi_{1}\right) \gg 1$ we can use the asymptotic property of the Bessel functions, viz. $I_{0}(x) \sim$ $I_{1}(x) \sim \mathrm{e}^{-x} / \sqrt{ }(2 \pi x)$ for $x \gg 1$. Physically, this corresponds to a $100 \%$ modulation of the particle current, i.e.

$$
J(t)=\langle J\rangle(1+\cos \omega t) .
$$

It may appear that validity of eqn (14) is in doubt because of two rather far-fetched assumptions that went into its derivation, namely that the variation of the barrier height is sinusoidal and that its amplitude is $50 \%$ of the mean value. However, the fact that the magnitudes of the coefficients at the first two harmonics are nearly equal (and this is all we need) follows from a much more general argument based on the duty cycle of the current. Indeed, for any periodic signal the amplitude of its first harmonic will be equal to the mean value provided the current flows in short pulses, i.e. provided the time during which the signal has an appreciable value is short compared to the period. In our case this means that the only requirement for the validity of eqn (14) is that $\Delta \psi_{1}(t)$ must be periodic and have an amplitude of several $k T / q$.

We can now estimate the power generated in the device by making the usual assumption that the barrier conductance $\sigma$ is related to the dc component $\langle I\rangle$ of the large signal total current in the same way as it is in the small signal theory. Using eqns (1a), (3)-(5) and (9) we find the following expression for the generated power, $P \equiv 1 / 2\langle I\rangle^{2}\left|R_{\text {tot }}\right|$

$$
P / S=\left(\frac{k T}{q}\right)^{2} \frac{\epsilon v_{s}}{2 \tilde{L}_{1}^{2}}\left[\frac{\cos \alpha}{1+\cos \alpha}-\frac{\omega \tilde{L}_{1}}{v_{s}} \operatorname{ctg} \alpha\right]
$$

where $S$ is the device area. Under optimal conditions, $\alpha \geqslant \pi / 3$, taking $\tilde{L}_{1}-50 \AA$, one finds that the second term in eqn (15) is unimportant for all but terahertz frequencies and that the power per unit area $P / S \sim$ $5 \times 10^{3} \mathrm{~W} / \mathrm{cm}^{2}$ is about an order of magnitude greater than in the conventional BARITT device[1]. The maximum area of a transit time device at a given frequency is usually limited by the requirement of matching its impedance with that of a cavity resonator. Simple estimates show that $S$ must be chosen so that $f^{2} S \sim c v_{s} / \kappa \sim$ $2.5 \times 10^{16} \mathrm{~cm}^{2} / \mathrm{sec}^{2}$, where $c$ is the speed of light and $\kappa \equiv \epsilon / \epsilon_{0}$ is the relative permittivity of the semiconductor. For $f \sim 100 \mathrm{GHz}$ we thus find $P \approx 10 \mathrm{~mW}$, and for other frequencies the power scales as $1 / f^{2}$.

In a separate publication it will be shown that the triangular barrier structure also offers the highest efficiency of any BARITT device designed to work at a given frequency. It is believed that studying this structure will help to establish the fundamental limit of efficiency for such devices.

Acknowledgement-We are grateful to S. M. Sze and B. C. DeLoach, Jr. for helpful comments.

\section{RETERENCES}

1. S. M. Sze, Physics of Semiconductor Devices, 2nd Edn, Chap. 10. Wiley-Interscience, New York (1981)

2. O. Eknoyan, E. S. Yang and S. M. Sze, Solid-St. Electron. 20, 291 (1977).

3. R. J. Malik, K. Board, C. E. C. Wood, L. F. Eastman, T. R. AuCoin and R. L. Ross, Electron. Lett. 16, 837 (1980).

4. R. Kazarinov and S. Luryi, to be published.

5. R. F. Kazarinov and S. Luryi, Appl. Phys. Lett. 38,810 (1981).

6. A. S. Tager, Soviet Phys. Uspekhi 9, 892 (1967).

7. D. Delagebeaudeuf, Rev. Techn. Thompson-CSF 1, 309 (1969).

8. D. Delagebeaudeuf and J. Lacombe, Electron. Lett. 9, 538 (1973). 